\title{
On telomeres long and short
}

\author{
Nathan Corbett, MSc; Martin Alda, MD
}

Telomeres and their shortening have been studied in the context of psychiatric disorders for about 10 years. A number of studies correlating telomere length (TL) with selected psychopathologies have generated intriguing and largely consistent findings: many psychiatric disorders are associated with shorter leukocyte TL. Yet, this observation can be interpreted in alternative ways, and choosing among them remains difficult owing to missing key pieces of information.

Telomeres are, essentially, caps on ends of chromosomes. They consist of TTAGGG repeats that usually become shorter with each successive cell division. Their main roles are to protect chromosomes from a gradual loss of nucleotides in dividing cells and to delineate DNA breakage from normal chromosome ends. They also halt cell division through activation of DNA damage recognition systems in response to acute cellular stress (e.g., radiation, oxidative stress), age (i.e., uncapped short telomeres) and disruption of various telomereassociated proteins (e.g., acquired or inherited mutations, cell signalling, toxins, drugs). ${ }^{1}$ Telomere integrity is maintained by a specialized polymerase system and various associated structural and signalling proteins; accordingly, the telomere system provides cells with information about their individual history and physiologic context.

The enzyme telomerase can elongate telomeres, but does not compensate fully for the cell division-related shortening in somatic cells. Telomerase is not substantially active in most adult somatic cells, with the exception of the germ line cells, proliferating leukocytes and endometrium and has a limited function in stem cells. Telomerase activity declines precipitously during the teen years, while TL declines most rapidly in early development, with subsequent slower attrition through the rest of the life course. ${ }^{1}$ Mean TL has been associated with 7 genetic loci that correspond with genes directly involved in telomere maintenance. ${ }^{2}$ Thus, we infer that TL results from the difference between factors negatively impacting TL on one hand and telomerase activity on the other. In humans, TL is heritable both generally ${ }^{3}$ and in specific cases of intergenerational telomere loss leading to genetic anticipation in a particular genetic disease. ${ }^{4}$ Telomere balance at any given time is a function of somatic growth rate, relevant genetics and epigenetics, age, environmental and physiologic context, psychological response to the environment, ${ }^{5}$ and physical or mental illness and/or its treatment, which define the rate of change in mean
TL at any given time. These factors combined with the heritability of TL "set points," which define early life (maximal) telomere length, determine somatic TL.

Populations vary dramatically in TL, even within regions, such as Europe, ${ }^{6}$ that have traditionally been considered relatively homogeneous and among people from different parts of the world. ${ }^{7}$ Likewise, TL varies among specific tissues within the same organism. This complexity is a good reminder that the telomere system is not amenable to simplistic analysis.

Telomere length is typically measured in leukocytes and referred to as leukocyte TL (LTL). Most commonly, LTL is estimated via a ratio of telomeric repeat amplification to that of a known single copy gene in multiplex quantitative polymerase chain reaction (qPCR) ${ }_{1}^{8}$ although other methods have better precision and cell-type and chromosome specificity; ${ }^{9}$ qPCR ratio does not describe the many telomeres of different lengths at every chromosome end in every cell or say anything about variance between cells or cell populations in the given sample. Given that very short telomeres occur randomly and with increasing probability at lower average TL, association between average TL and biomolecular reality is, at best, probabilistic. In addition, the normal variation in human average TL is substantial, necessitating large cohorts to achieve sufficient statistical power for inference testing. While current techniques are sufficient for high-level descriptive work, chromosome resolution, longitudinal "telomere velocity" studies will provide more useful information.

Among psychiatric disorders, LTL has been found to be significantly reduced in patients with bipolar disorder, psychosis, major depression and anxiety disorders. Shorter telomeres are commonly thought to reflect - or result from accelerated aging processes. One proposed mechanism involves oxidative stress, negative impact of reactive oxygen species and impaired mitochondrial function. The same mechanisms may, for instance, play a role in the pathophysiology of bipolar disorder and/or its progression, with recent results indicating that long-term lithium therapy leads to a detectable relative increase in LTL versus controls. ${ }^{10}$ There is clearly much to learn in this regard, as lithium therapy has not been shown to modulate leukocyte telomerase function. ${ }^{11}$

Most observations of telomere shortening in psychiatry come from investigations of leukocytes, not brain tissue. Few studies examined TL in postmortem brains. Teyssier and

Correspondence to: M. Alda, Department of Psychiatry, Dalhousie University, 5909 Veterans' Memorial Lane, Halifax NS B3H 2E2; malda@dal.ca

J Psychiatry Neurosci 2015;40(1):3-4.

DOI: 10.1503/jpn.140347 
colleagues ${ }^{12}$ reported no difference between patients with depression and healthy controls. On the other hand, Sequeira and colleagues ${ }^{13}$ found telomere shortening in the hippocampi, but not the dorsolateral prefrontal cortex, of individuals with depression. Also, age-dependent TL reduction was present only in some brain regions..$^{13}$ By taking a different approach, Szebeni and colleagues ${ }^{14}$ found TL shortening in white matter oligodendrocytes in major depression. In aggregate, brain cells have low levels of telomerase activity comparative to proliferative tissues, such as bone marrow or mucosa, and different brain regions differ in cell type composition, having variable ratios of neurons and glial cells, which have distinct telomere dynamics due to categorically different rates of cell turnover. Shorter telomeres in stem cells may impair neurogenesis, ${ }^{15}$ a mechanism hypothesized as relevant to the pathophysiology of mood disorders. Finally, a single study in Alzheimer disease found no correlation between LTL and brain $\mathrm{TL}_{1}^{16}$ though LTL has been associated with volumes of the relevant brain regions ${ }^{17}$ and with disease status.

The LTL in many psychiatric disorders appears to follow a dose-response pattern whereby more severe and more chronic conditions were associated with shorter telomeres, which has been demonstrated both in depression ${ }^{5}$ and psychosis. ${ }^{18}$ This observation is consistent with the fact that these disorders and their progression are frequently associated with physical conditions that are also associated with shorter telomeres, such as metabolic syndrome or diabetes mellitus. Patients with these comorbid conditions are known to have poorer psychiatric outcomes. ${ }^{19}$

In summary, we found a number of consistent and replicated findings, but their interpretation is not easy. A common view is that shorter telomeres reflect premature aging in psychiatric patients, and this aging is manifest in mitotically active cells. Telomere shortening then would result from the same pathophysiological processes (or allostatic factors involved in their etiology) affecting the body and the brain; for instance it could be a consequence of metabolic and inflammatory changes associated with the progression of psychiatric disorders. But several important questions remain. First, the existing data do not allow us to determine if telomere shortening is present in all patients with major psychiatric disorders or whether it plays a role in only subtypes of disorders associated with inflammation and oxidative stress..$^{20}$ Also, we do not know if the TL and/or propensity for telomere shortening are associated with the predisposition to major psychiatric disorders. To answer this question, we may need a prospective analysis of TL in people at genetic risk for such disorders. Finally, it will be important to clarify the relevance of partial reversibility of pathological TL changes by treatment, for instance with lithium. ${ }^{10}$ The effect of lithium is intriguing and appears to parallel its neuroprotective action. But similar to neuroprotective effects, it is not clear how essential it is for therapeutic response.

While telomeres present an intriguing window into the cellular perspective on allostasis, the inherent complexity of this system warrants caution. Considerable resources have been expended in developing a robust literature in telomere science, largely thanks to the prominent role of telomeres in cancer biology, and it will be crucial to properly leverage this body of basic research to design and implement responsible research studies that clarify rather than confuse our understanding.

Affiliations: Faculty of Medicine (N. Corbett) and Department of Psychiatry (M. Alda), Dalhousie University, Halifax, Nova Scotia, Canada.

Competing interests: None declared.

\section{References}

1. Aubert G, Lansdorp PM. Telomeres and aging. Physiol Rev 2008;88: 557-79.

2. Codd V, Nelson CP, Albrecht E, et al. Identification of seven loci affecting mean telomere length and their association with disease. Nat Genet 2013;45:422-7.

3. Njajou OT, Cawthon RM, Damcott CM, et al. Telomere length is paternally inherited and is associated with parental lifespan. Proc Natl Acad Sci U S A 2007;104:12135-9.

4. Vulliamy T, Marrone A, Szydlo R, et al. Disease anticipation is associated with progressive telomere shortening in families with dyskeratosis congenita due to mutations in TERC. Nat Genet 2004;36:447-9.

5. Epel ES, Blackburn EH, Lin J, et al. Accelerated telomere shortening in response to life stress. Proc Natl Acad Sci U S A 2004;101:17312-5.

6. Eisenberg DT, Salpea KD, Kuzawa CW, et al. Substantial variation in GPCR measured mean blood telomere lengths in young men from eleven European countries. Am J Hum Biol 2011;23:228-31.

7. Rehkopf DH, Dow WH, Rosero-Bixby L, et al. Longer leukocyte telomere length in Costa Rica's Nicoya Peninsula: a populationbased study. Exp Gerontol 2013;48:1266-73.

8. Cawthon RM. Telomere length measurement by a novel monochrome multiplex quantitative PCR method. Nucleic Acids Res 2009;37:e21.

9. Aubert G, Hills M, Lansdorp PM. Telomere length measurementcaveats and a critical assessment of the available technologies and tools. Mutat Res 2012;730:59-67.

10. Martinsson L, Wei $\mathrm{Y}, \mathrm{Xu} \mathrm{D}$, et al. Long-term lithium treatment in bipolar disorder is associated with longer leukocyte telomeres. Transl Psychiatry 2013;3:e261.

11. Soeiro-de-Souza MG, Teixeira AL, Mateo EC, et al. Leukocyte telomerase activity and antidepressant efficacy in bipolar disorder. Eur Neuropsychopharmacol 2014;24:1139-43.

12. Teyssier J-R, Ragot S, Donzel A, et al. Telomeres in the brain cortex of patients with major depressive disorder. Act Nerv Super 2014;56:89-94.

13. Sequeira A, Mamdani F, Vawter M, et al. Variable telomere length across post-mortem human brain regions and specific reduction in the hippocampus of major depression. XXIInd World Congress of Psychiatric Genetics 2014. Available: http://2014.ispg.net/wp -content/uploads/2014/10/Oral-Abstract-Book-Updated-10-13-14 .pdf (accessed 2014 Nov. 18).

14. Szebeni A, Szebeni K, DiPeri T, et al. Shortened telomere length in white matter oligodendrocytes in major depression: potential role of oxidative stress. Int J Neuropsychopharmacol 2014;17:1579-89.

15. Ferrón SR, Marques-Torrejon MA, Mira H, et al. Telomere shortening in neural stem cells disrupts neuronal differentiation and neuritogenesis. J Neurosci 2009;29:14394-407.

16. Thomas P, O'Callaghan NJ, Fenech M. Telomere length in white blood cells, buccal cells and brain tissue and its variation with ageing and Alzheimer's disease. Mech Ageing Dev 2008;129:183-90.

17. King KS, Kozlitina J, Rosenberg RN, et al. Effect of leukocyte telomere length on total and regional brain volumes in a large population-based cohort. JAMA Neurol 2014;71:1247-54.

18. Martinez D, Rizzo L, Silva P, et al. Investigation of telomere length in different stages of schizophrenia. XXIInd World Congress of Psychiatric Genetics 2014. Available: http://2014.ispg.net/wp -content/uploads / 2014/10/Poster-Abstract-Book-Updated-with -Cover-10-13-14.pdf (accessed 2014 Nov 18).

19. Ruzickova M, Slaney C, Garnham J, et al. Clinical features of bipolar disorder with and without comorbid diabetes mellitus. Can J Psychiatry 2003;48:458-61.

20. Garcia-Rizo C, Fernandez-Egea E, Miller BJ, et al. Abnormal glucose tolerance, white blood cell count, and telomere length in newly diagnosed, antidepressant-naive patients with depression. Brain Behav Immun 2013;28:49-53. 